\title{
An Integral Approach to Multi-physics Application for Packed Bed Reactors
}

\author{
Bernhard Peters ${ }^{\mathrm{a}^{*}}$, Xavier Besseron ${ }^{\mathrm{a}}$, Alvaro Estupinan ${ }^{\mathrm{a}}$, Florian Hoffmann ${ }^{\mathrm{a}}$, \\ Mark Michael $^{\mathrm{a}}$, Amir Mouhmadi ${ }^{\mathrm{a}}$, and Mohammad Mohseni ${ }^{\mathrm{a}}$ \\ ${ }^{a}$ University of Luxembourg, 6, rue Coudenhove-Kalergi, L-1359 Luxembourg, \\ Luxembourg \\ bernhard.peters@uni.lu
}

\begin{abstract}
A large number of engineering applications involve granular material or a particulate phase in combination with a gaseous or liquid phase. Predominant applications are as diverse as pharmaceutical industry e.g. drug production, agriculture food and processing industry, mining, construction and agricultural machinery, metals manufacturing, energy production and systems biology. Common to all these application is that they cover a large spectrum of length scales ranging from inner particle length scales to global dimensions of the reactor. In order to describe the processes and their interaction accurately, tailored algorithms are required for prediction and analysis.

The current numerical approach of the Extended Discrete Element Method (XDEM) is based on an Eulerian-Lagrange coupling. For this purpose the solid phase consisting of individual particles is treated by the Lagrange method that describes both the dynamic state i.e. position and orientation of each particle in space and time and its thermodynamic state e.g. internal temperature and species distribution. The flow of gas in the void space between the particles is predicted by traditional and well-proven Computational Fluid Dynamics (CFD) taking into account heat and mass transfer between the particles and the surrounding gas phase. Hence, the entire process represented by the sum of all particle processes in conjunction with fluid dynamics.

The afore-mentioned numerical concept was applied to predict pyrolysis of a packed bed of wood particles in a cylindrical reactor. A comparison of predicted results with experimental data show good agreement. Hence, the numerical concept is able to resolve a large range of length scales for solid reaction engineering. An analysis of detailed results helps to uncover the underlying physics of the process, and thus, allows for an improved design and operation conditions.
\end{abstract}

Keywords: Extended Discrete Element Method, numerical modelling, multi-physics

\section{Introduction}

Contrary to continuum models an alternative approach considers the solid phase as discrete, while the flow of liquids or gases is treated as a continuum phase in the void space between the particles, and therefore, is labelled the Combined Continuum and Discrete Model (CCDM) as employed by Tsuji et al. (1993), Hoomans et al. (1996), and $\mathrm{Xu}$ and Yu (1997, 1998). Due to a discrete description of the solid phase, constitutive relations are omitted, and therefore, leads to a better understanding of the fundamentals. This was also concluded by Zhu et al. $(2007,2008)$ during a review on particulate flows modelled with the CCDM approach. Based on this approach Sheng et al. (2013) 
investigated into the micro-scale heat transfer of packed beds and micro-fibrous entrapped catalysts and concluded that the thermal resistance of the contact points account for more than $90 \%$ of the total resistance. Similarly, Kon et al. (2013) modelled the liquid flow in the lower part of a blast furnace by the MPS method. CCDM has seen a mayor development in last two decades and describes motion of the solid phase by the Discrete Element Method (DEM) on an individual particle scale and the remaining phases are treated by the Navier-Stokes equations.

However, current CCDM approaches should be extended to a truly multi-phase flow behaviour as opposed to the Volume-of-Fluid method and the multi-phase mixture model stated by Sheng (2013). Furthermore, particle shapes other than spherical geometries have to be taken into account to meet engineering needs according to Zhu et al. $(2007,2008)$. This efforts should ideally be complemented by poly-disperse particle systems as employed by Peters and Dziugys (2011). All these efforts should contribute to a general link between continuum and discrete approaches so that results are quantified for process modelling as applied by Peters et al. $(2013,2014)$.

\section{Numerical Approach}

A novel technique referred to as Extended Discrete Element Method (XDEM) (Wikipedia, 2012, Peters, 2013) has emerged only recently that offers a significant advancement for multi-physics applications. It is based on a coupled discrete and continuous i.e. Lagrange-Euler simulation concept. XDEM treats the solid phase representing the particles and the fluid phase or a structure as two distinguished entities that are coupled through heat, mass and momentum transfer. An outstanding feature of the numerical concept is that each particle in addition to its position and orientation in time and space is described by its thermodynamic state e.g. temperature and reaction progress. The thermodynamic state is described by one-dimensional and transient differential conservation equations for mass, energy and momentum for each individual particle and thus, characterizes the overall behaviour of the reactor as the sum of all particle processes. Predicted results for all individual particles allow a detailed analysis of the reaction process.

Through predicting position and orientation of all particles their arrangement in space is known that determines the 3-dimensional distribution of void space between the particles. This essentially represents a porous structure through which the fluid e.g. liquid or gas streams. The fluid is in contact with the surface of the particles and determines heat and mass exchange between the particle's surface and the fluid i.e. temperature and composition in the vicinity of the particle. In order to predict flow through the interstitial space a classical CFD approach is preferred for which the software framework of OpenFoam is employed. Its library offers a large selection of solves for different applications and allows as an open-source software a tailored development of solvers suiting the needs of the above-mentioned applications.

Hence, the proposed methodology provides a high degree of resolution ranging from scales within a particle to the continuum phase as global dimensions and offers superior features as compared to traditional and pure continuum mechanics approaches. The latter does not include detailed information on a particulate scale that has to be compensated for by empirical correlations such as distribution of void space in a packed or moving bed. For a more detailed description the reader is referred to Peters and Dziugys. 


\section{Results}

This concept was applied to predict drying and pyrolysis of spruce wood particles in a packed bed of which the properties are given in the following table 1.

Table 1. Properties of spruce wood

\begin{tabular}{ll}
\hline Property & Value \\
\hline Density & $450 \mathrm{~kg} / \mathrm{m}^{3}$ \\
\hline Specific heat & $(1500.0+\mathrm{T}) \mathrm{J} / \mathrm{kgK}$ \\
\hline Conductivity spruce & $0.35 \mathrm{~W} / \mathrm{mK}$ \\
\hline Conductivity char & $0.1 \mathrm{~W} / \mathrm{mK}$ \\
\hline Porosity & 0.68 \\
\hline Pore diameter & $5010^{-6} \mathrm{~m}$ \\
\hline
\end{tabular}

For an efficient validation process as described by Peters and Smula-Ostaszewska (2010), predicted results were first compared to measurements of a single particle for which Gronli (1996) carried out pyrolysis experiments of spruce cylinders. One of the circular side surfaces was exposed to a radiative flux of $80.0 \mathrm{~kW}$ and $130.0 \mathrm{~kW}$, respectively. The temperatures were recorded at 5 different positions $(\mathrm{L}=1,4,8,12,24$ $\mathrm{mm}$ ) along the cylinder axis during an experiment. Minimum and maximum temperatures at different positions were evaluated under identical conditions for several experiments so that experimental uncertainty related to the unavoidably difference in wood samples was obtained. Thus, an improvement of predictions within the experimental error is not necessary. For a more detailed description of the experimental set-up and pyrolysis model the reader is referred Gronli (1996).

Figues 1 and 2 depict a comparison between the measured temperature and the predictions at different locations within the sample.

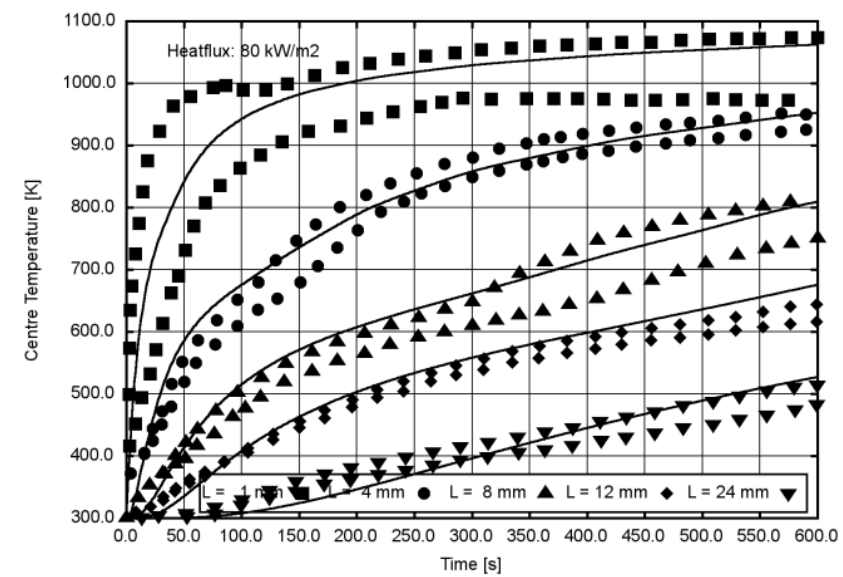

Figure 1. Comparison between measured and predicted interior temperatures during pyrolysis of a cylindrical spruce wood sample at a radiative heat flux of $80.0 \mathrm{~kW} / \mathrm{m}^{2}$

In general, the temperature profiles attain the exponential characteristics of heat conducted within a solid material (Carslaw, M. S. and Jaeger, J. C., 1959). The measured maximum and minimum temperatures express differences of more than $100 \mathrm{~K}$ 


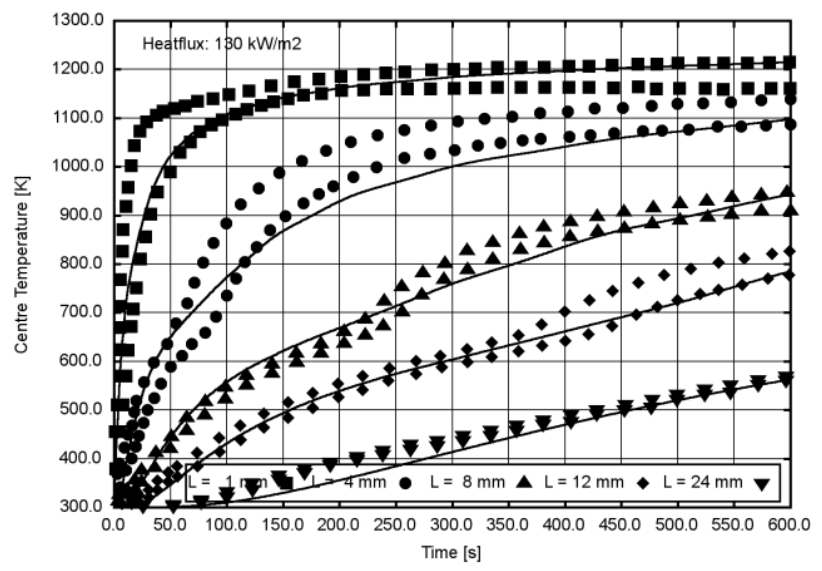

Figure 2. Comparison between measured and predicted interior temperatures during pyrolysis of a cylindrical spruce wood sample at a radiative heat flux of $130.0 \mathrm{~kW} / \mathrm{m}^{2}$

during its evolution for the temperatures measured closest to the sample surface. However, these differences reduce to app. 30 - 50 K for inner locations of temperature measurements. The predicted transient temperature profiles at the 5 locations fall mostly between the maximum and minimum measured temperature profiles, and thus, agree well with experiments.

After a successful validation of pyrolysis for a single particle, the model with its kinetic parameters was applied to each individual particle of a packed bed in a cylindrical reactor depicted in Figure 3. Hot inert gas streamed through the reactor to heat-up the particles and to provide sufficient heat for pyrolysis. A detailed description of the experimental set-up is found in Schröder (1999).

Figure 3 shows the rather inhomogeneous pyrolysis

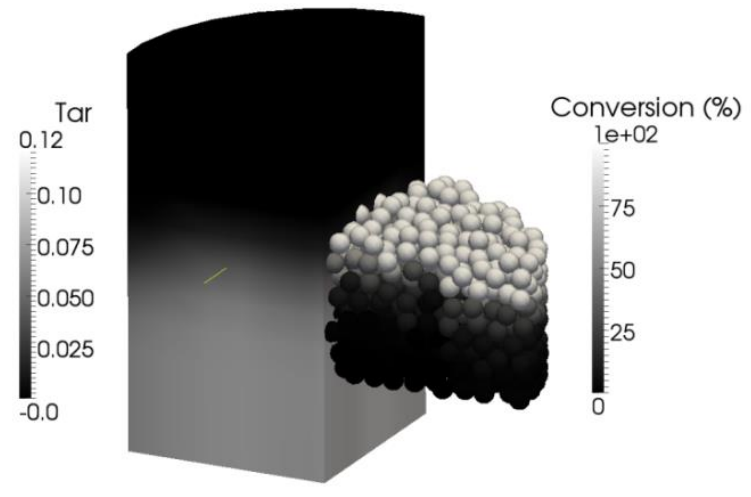

Figure 3. Predicted conversion rates of individual particles during pyrolysis in a packed bed in conjunction with the tar distribution in the gas phase

process taking place in the upper part of the reactor where the hot enters the void space of the packed bed. Rather advanced pyrolysis is observed for particles near the circumference of the reactor as compared to particles located at the centre which is due to higher temperatures around the inner reactor wall. The latter is due to increased porosity in conjunction with higher mass flow rates than in the centre of the reactor, and thus, augmented heat transfer and pyrolysis rate.

Furthermore, Figure 4 depicts the spatial and temporal distribution of temperature for two particles located at the top and bottom of the reactor. Although the particles do not experience a spatial gradient for temperature due to a slow convective heat transfer from 
the hot in-flow of gas, a spatial resolution is in general required for particles of larger size or high heating rates, for which significant temperature gradients within the particles develop. These temperature differences affect the reaction progress to a large extent and cannot be described with a space-averaged mean of the particle temperatures. Furthermore, the heat-up process for the bottom particle for the right sub-figure in Figure 4 is significantly delayed due to an earlier heat-up of the particles in the upper part of the reactor where the hot incoming air transfers its heat much earlier to these particles as to be seen in the left sub-figure of Figure 4. Once the hot incoming gas has transferred its heat to the upper particles, the gas temperature decreases and consequently, particles in the lower part of the reactor experience a reduced heating rate in conjunction with a delay of app. 1000 s. Hence, the Extended Discrete Element Method offers results with a high degree of resolution in both time and space of which the analysis contributes significantly to the understanding of the underlying physics. Additionally, many empirical correlations such as the distribution of void space in a reactor are omitted, and thus reduce experimental work to a large extent.

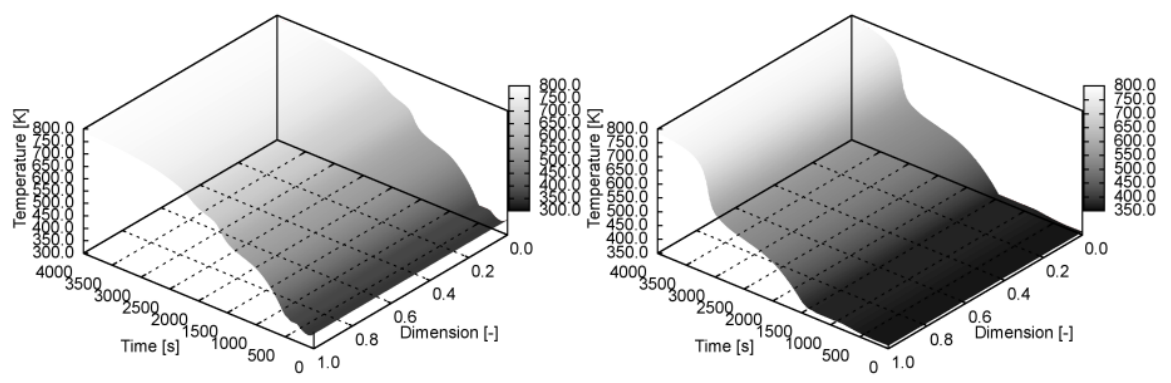

Figure 4. Predicted conversion rates of individual particles during pyrolysis in a packed bed in conjunction with the tar distribution in the gas phase

\section{Conclusions}

The current contribution describes the Extended Discrete Element Method (XDEM) that is applied to thermal conversion of packed beds as often encountered in process engineering. The methodology relies on a Lagrange-Eulerian approach that couples effectively the particulate phase with a gas streaming through the void space of a packed bed reactor. The particle processes of the packed bed are described by the solution of one-dimensional and transient differential conservation equations for mass and energy. This set of equation is solved individually for each particle of the packed bed by fast and efficient algorithms. Thus, the thermodynamic state of each particle is determined taking into account space and time-dependent boundary conditions prevailing within the reactor such as heat and mass transfer between the particle surface and the surrounding gas phase. The latter is described by solving the conservation equations of classical Computational Fluid Dynamics (CFD). Hence, the numerical concept provide results over a large range of length scales ranging from inner particles processes to the global dimensions of the reactor. The presented approach deals with poly-disperse particle shapes and takes heat and mass transfer between the particles' surface and the surrounding gas phase into account that distinguishes the presented concept from current approaches. Thus, physics are described to a fine degree that allows an in-depth analysis of obtained results. It unveils the underlying physics of the processes involved 
and as a computer-aided tool contributes significantly to an improved design and operating conditions.

\section{Acknowledgement}

This work was funded by the Fond National de la Recherche Luxembourg.

\section{References}

Tsuji, Y., T. Kawaguchi, T. and Tanaka, T., Discrete particle simulation of two- dimensional uidized bed. Powder Technol., 77(79), 1993.

Hoomans, B. P . B., Kuipers, J. A. M., Briels, W. J. and Van Swaaij, W. P. M., Discrete particle simulation of bubble and slug formation in a two-dimensional gas-uidized bed: A hard-sphere approach. Chem. Eng. Sci., 51, 1996.

$\mathrm{Xu}, \mathrm{B} . \mathrm{H}$. and $\mathrm{Yu}, \mathrm{A}$. B., Numerical simulation of the gas-solid ow in a fluidized bed by combining discrete particle method with computational fluid dynamics. Chemical Engineering Science, 52:2785, 1997.

$\mathrm{Xu}, \mathrm{B}$. H. and $\mathrm{Yu}, \mathrm{A}$. B., Comments on the paper numerical simulation of the gas-solid flow in a fluidized bed by combining discrete particle method with computational fluid dynamics-reply. Chemical Engineering Science, 53:2646-2647, 1998.

Zhu, H. P., Zhou, Z. Y., Yang, R. Y. and Yu, A. B., Discrete particle simulation of particulate systems: Theoretical developments. Chemical Engineering Science, 62:3378 \{3396, 2007. 1

Zhu, H. P., Zhou, Z. Y. , Yang, R. Y. and Yu, A. B., Discrete particle simulation of particulate systems: A review of major applications and findings. Chemical Engineering Science, 63:5728-5770, 2008.

Sheng, M., Gonzalez, C., Yantz, W., Cahela, D,. Yang, H., Harris, D. and Tatarchuk, B.. Microscale heat transfer comparison between packed beds and micro-fibrous entrained calalysts. Engineering Applications of Computational Fluid Mechanics, 7(4):47-485, 2013.

Kon, T., Natsui, S. ,Shin, K., Ueda, S.,Inoue, R.and Ariyama, T. Modelling of liquid flow in the lower part of blast furnace by mps method. In The $5^{\text {th }}$ International Conference STEELSIM 2013, Sptember 10 - 12, 2013, 2013.

Wang, C. Y., Tramsport Phenomena in Porous Media, chapter Modelling Multi- phase Flow and Transport in Porous Media. Oxford Pergamon, 1998.

Peters, B. and Dzuigys, A., A discrete approach to model thermal conversion of beds of solid fuels by the discrete particle method (DPM). In ESCAPE21 - 21st European Sympo- sium on Computer-Aided Process Engineering, Porto Carras Resort, Chalkidiki, Greece, May 29 - June 1, 2011, 2011.

Wikipedia, 2012, Extended discrete element method en.wikipedia.org/wiki/Xdem, accessed 13.09.12

Peters, B., 2013, The extended discrete element method (XDEM) for multi-physics applications, Scholarly Journal of Engineering Research, 2(1):1-20, 2013

Peters, B. and Smula-Ostaszewska, J., Evaluation of kinetic data and modelling of sulphur dioxide during combustin of switcgrass, 20 th European Symposium on Computer Aided Process Engineering, Elsevier, 2010

Peters, B., The extended discrete element method (XDEM) for multi-physics applications. Scholarly Journal of Engineering Research, 2(1):1-20, 2013

A.H. Mahmoudi, F. Hoffmann, and B. Peters. Application of xdem as a novel approach to predict drying of a packed bed. International Journal of Thermal Sciences, 75:65-75, 2014

Gronli M., A theoretical and experimental study of the thermal degradation of biomass, $\mathrm{PhD}$ thesis, NTNU Trondheim, 1996

Carslaw, M. S. and Jaeger, J. C., Conduction of Heat in Solids. Oxford University Press, 1959

Schröder, E., Bestimmung des Druckverlustes und des Wärmeüberganges von gasdurchströmten Feststoffschüttungen in der PANTHA Anlage, Forschungszentrum Karlsruhe, FZKA 6373, 1999 